\title{
Determination of Bubble Size Distribution using Ultrasound Array Imaging
}

\author{
${ }^{1 *}$ Marcus Ingram, ${ }^{1}$ Carmelo Mineo, ${ }^{1}$ Anthony Gachagan, ${ }^{2}$ Anthony J. Mulholland, ${ }^{3}$ Alison Nordon and ${ }^{4}$ Martin \\ Hegarty, ${ }^{1}$ Centre for Ultrasonic Engineering, University of Strathclyde, Glasgow, UK, ${ }^{2}$ Dept. Engineering \\ Mathematics, University of Bristol, Bristol, UK, ${ }^{3}$ Dept. Pure and Applied Chemistry, University of Strathclyde, \\ Glasgow, UK, ${ }^{4}$ BP Chemicals Ltd, Hull, UK, *m.ingram@strath.ac.uk
}

\begin{abstract}
In this paper, ultrasonic phased arrays are deployed as an imaging tool for industrial process analysis. Such arrays are typically used for sonar, medical diagnosis and non-destructive testing, however, they have not yet been applied to industrial process analysis. The precise positioning of array elements and high frequencies possible with this technology mean that highly focused images can be generated that cannot currently be achieved using ultrasound tomography. This paper aims to highlight the potential of this technology for measurement of bubble size distribution (BSD) and to demonstrate its application to both intrusive and non-invasive process measurement. Ultrasound images of bubble reflectors are generated using the total focusing method deployed using a 32 element, $5 \mathrm{MHz}$ linear phased array and an image processing algorithm for BSD determination is presented and evaluated under stationary and dynamic acquisition conditions. It is found that the sizing accuracy is within $10 \%$ for stationary reflectors larger than $4 \lambda$ in diameter and that the algorithm is stable across the expected spatial variation of reflectors. The phased array is coupled to a six-axis robotic arm to scan a solid sample containing bubble reflectors at velocities up to $500 \mathrm{mms}^{-1}$. The sizing accuracy is within $45 \%$ for bubbles larger than $4 \lambda$ in diameter and at velocities up to $300 \mathrm{mms}^{-1}$. However, above this velocity the algorithm breaks down for reflectors smaller than $9 \lambda$ in diameter. The ultrasound system is applied to a stream of air bubbles rising through water that is verified via photographic analysis. Images were generated both intrusive and non-invasive, via a $10 \mathrm{~mm}$ Perspex barrier, to the process stream. The high bubble density in the process stream introduced scattering, limiting the measurement repeatability and the sample size in the measured distribution. Notwithstanding, this result demonstrates the potential of this technology to size bubbles for intrusive and non-invasive process analysis.
\end{abstract}

Index Terms-ultrasound array imaging, total focusing method, industrial process analysis, bubble size distribution, noninvasive imaging

\section{INTRODUCTION}

This paper presents a novel methodology for the determination of the bubble size distribution (BSD) in a two-phase industrial flow. The BSD is determined from highly focused images of the process generated using an ultrasonic phased array. An algorithm to process these images is presented that determines the diameter of bubble reflectors captured in each image frame, whereupon the BSD can be estimated using multiple image frames.

Engineering and Physical Sciences Research Council (grant number EPM5076471)
Recent technological advances in multiple-channel phased array controllers (PAC) and general purpose graphics processing units (GPGPU) have accelerated ultrasonic data acquisition and image construction algorithms [1]. Together, these have created the opportunity to apply phased array imaging towards dynamic industrial processes that require data to be quickly recorded and interpreted for real-time process analysis [2].

Ultrasonic phased arrays are typically deployed for imaging into optically opaque materials, for example, tissue in medicine and steel in non-destructive testing [3]. Ultrasonic phased array hardware is relatively cheap to maintain and it provides a safe, non-ionising methodology for imaging [4]. Ultrasound imaging has a significant advantage over optical based imaging techniques because it does not require an optical path to exist between the sensor and the image target. This means ultrasound measurement is not necessarily confined to optically transparent processes or vessel materials [5]. This is because ultrasound wave propagation is dependent on the mechanical properties of the propagating medium [6].

Ultrasonic spectroscopy is an active measurement technique used for particle sizing in process analysis [7]. This involves quantification of the scattering parameters of the propagating medium, for example, the attenuation and backscatter coefficients [7]. As such, the technique requires a good understanding of the physical constants relating to the measured system, for example, its thermal conductivity, density and elastic modulus [7]. Due to the wide frequency range feasible, from $20 \mathrm{kHz}$ to $200 \mathrm{MHz}$, particle sizing between $10 \mathrm{~nm}$ and 1 $\mathrm{mm}$ is possible [8]. This makes ultrasonic spectroscopy highly suitable for typical colloidal and emulsion processes [9]. On the other hand, at the boundary between a gas and a liquid, any ultrasound energy injected into the system is strongly reflected back towards the transducer due to the large difference in acoustic impedance. This means that gas-liquid systems are well-suited for pulse-echo ultrasound imaging.

Gas-liquid two-phase systems are found in a wide variety of industrial processes. For example, solvent sublation is used for waste water treatment whereby hydrophobic organic compounds adsorb at the bubble-fluid interface removing organic pollutants [10]. In the petrochemical industry, the FischerTropsch process is used to convert natural gas into commercial products like fuels [11]. During this process syngas bubbles are contacted with liquid slurries containing catalyst particles. These bubbles can be on the millimetre length scale and travel at low superficial gas velocities within the fluid to 
maximise the interfacial contact time [11]. Coal liquefaction is also used by the petrochemical industry; hydrogenation of the hydrocarbon is performed by supplying hydrogen bubbles to a coal slurry [12]. Bubbles passing through a fluid are also used in separation processes, for example, in flotation cells [13]. Suspended molecules adsorb to bubbles rising to the surface of a fluid and characterisation of the froth provides an indication of the drainage and coalescence behaviour inside the flotation cell [14].

Measurement of the BSD is key to the characterisation of these processes. The BSD provides an indication of the total surface area of bubbles in a two-phase flow [15]. To characterise the efficiency of mass transfer between two phases requires a strong understanding of this interfacial area [15]. However, in the context of a typical industrial process environment there are several challenges associated with its measurement. Current technologies for the determination of BSD are often intrusive or disruptive to the process flow, for example, using wire-meshes to measure the fluid conductivity to infer the BSD [16] [17]. On the other hand, non-invasive techniques, such as photographic analysis, require a clear optical path to exist between the measurement sensor and the process [18].

To extract spatial information from process images, it is necessary to convert the dimensions of identified objects from units of pixels to millimetres. For optical images acquired with a digital camera, the image construction process is intrinsic to the camera device. This means the end-user does not define the pixel dimension and this must be determined experimentally [19]. For example, a calibration grid can be positioned in the test cell and images acquired under identical experimental conditions can be used to determine the pixel dimension [2]. This has been specifically highlighted as a drawback to using optical images for industrial process analysis [15]. On the contrary, ultrasonic image construction is not limited in this sense as the pixel dimension is user-defined prior to image construction, for example, when using the total focusing method (TFM) [20]. Therefore, square pixels of known dimension can be used, greatly reducing experimental uncertainty associated with the conversion of object sizes from pixels to millimetres. Another technical challenge associated with optical images is that objects of interest can be outof-focus. This reduces the accuracy of object edge detection leading to inaccurate bubble sizing [21]. This presents another advantage of using ultrasound imaging for process analysis; images generated using the TFM are, by definition, focused at every pixel.

Currently, the extent of ultrasound imaging for process analysis is limited to ultrasound transmission tomography [5]. This involves pitch-catch ultrasound imaging, whereby the ultrasound path length is determined by the diameter of the process vessel and the density of reflectors within the process. Therefore, ultrasound transmission tomography typically uses low megahertz frequency signals to avoid significant signal attenuation [22], however, this is at the cost of reduced lateral resolution. An advantage of ultrasound transmission tomography over pulse-echo phased array imaging is that the received signals contain contributions from both reflection and transmission events within the process instead of relying purely on the reflected signal amplitude. For this reason, pulse-echo imaging is more suited for imaging close to the transducer or at the periphery of the process and ultrasound transmission tomography is more suitable for imaging of the centre of the process vessel. The reduced path length means higher frequencies can be deployed using phased arrays, increasing the lateral resolution of the ultrasound images. Overall, the two technologies exhibit the same advantages with respect to non-invasive process measurement; they should be considered as complementary technologies.

Given the recent technological advancements, it is the aim of this paper to demonstrate the application of ultrasonic phased array imaging for industrial process analysis. To highlight their potential applicability, it is also the aim to show how these images can be used to determine the BSD of a dynamic process. In doing so, the accuracy of the process measurement is discussed and the challenges associated with extending this technology to an industrial plant are presented.

The paper is organised as follows. In Section II the ultrasound system is described and the experimental materials are presented. In Section III, the ultrasound image processing algorithm is demonstrated for the determination of bubble diameter from ultrasound images. Following this, in Section IV, a second image processing algorithm is briefly described using photographic images as the input. This second image processing algorithm is used to verify the findings from the ultrasonic image analysis. Section $\mathrm{V}$ presents the results from the application of the ultrasound image processing algorithm to images of bubble reflectors acquired under stationary and dynamic conditions. A discussion on the potential of this technology for industrial process analysis is presented in Section VI and finally the paper ends with conclusions in Section VII.

\section{Experimental ApPARATUS AND Methods}

\section{A. Ultrasound Hardware and Software}

The ultrasonic hardware consisted of a 128-element linear phased array with a $5 \mathrm{MHz}$ centre frequency (Vermon SA, France) and an FIToolbox PAC (Diagnostic Sonar Ltd, UK). The phased array had an element pitch of $0.7 \mathrm{~mm}$ corresponding to approximately one wavelength in Perspex. As a result, beam steering and focussing capabilities were reduced, however, the sensitivity of the probe was enhanced by the larger element width.

In this paper, the phased array is deployed using full matrix capture (FMC) [20]. By transmitting each element in the aperture individually and receiving on all elements, this procedure records every transmit-receive combination for each element location, corresponding to the maximum quantity of information about the image scene. The FMC dataset can be reconstructed into a highly focused image using the TFM [20]. The image construction time is a function of the number of pixels in the image scene and this is user-defined. The TFM script used in this paper takes advantage of GPGPU technology (GeForce GTX $750 \mathrm{Ti}$, NVIDIA, USA) to enable faster image construction [23]. In addition to the enhanced image 
TABLE I: FMC acquisition rate when fired $20 \mathrm{~mm}$ into water at the maximum pulse repetition frequency.

\begin{tabular}{cc}
\hline Active Aperture [Elements] & FMC Acquisition Rate [Hz] \\
\hline 32 & 145 \\
64 & 37 \\
128 & 9 \\
\hline
\end{tabular}

construction rate, this algorithm can incorporate refraction at an acoustic boundary, for example, between the process vessel wall and fluid, by specifying the acoustic velocity in the two media and the thickness of the first layer. The refracted timeof-flight between the array elements and the focal point is determined using a classical minimum search with respect to Fermat's principle to find the shortest path length [23]. These specific advantages of the GPGPU TFM imaging algorithm and the beamforming constraints imposed by the phased array design led to the application of FMC for the array deployment.

The PAC consisted of 32 transmit-receive channels such that multiplexing across the channels was required to deploy an active aperture greater than 32 elements. The time required to perform multiplexing significantly reduced the acquisition rate for a given image depth, shown in Table I. Therefore, given the dynamic nature of the image target, a 32 element aperture was selected to maximise the FMC acquisition rate.

All FMC data in this paper have been filtered to reduce noise artefacts using a zero-phase Butterworth band-pass filter prior to TFM image construction. The lower and upper bandstop frequencies were set to $\pm 2 \mathrm{MHz}$ of the probe's designed centre frequency. It was important not to introduce phase shifts during the filter process because the time domain response is used for the TFM image construction. This was achieved by applying the filter, reversing the order of the time samples, reapplying the filter and reversing the time domain samples back to their initial order to recover the filtered amplitude but with the original phase.

To reduce the impact of spatial side-lobe activity on the TFM images, the 32 element aperture has been apodised with respect to the transmitting element during TFM image construction using a Hamming window. The window was designed using the number of elements in the aperture as its input, where each index of the window corresponds to a transmitting element. Next, for a given transmit, all the received signals were weighted with respect to the value of the Hamming window at the index position corresponding to the transmitting element. Time-gain compensation has not been applied during image construction because the samples investigated in this work were not highly scattering, so signal attenuation was not considered significant. Also, envelope detection has not been applied because this led to spurious segmentation of the TFM images in Section III.

All TFM images in this work were generated using square pixels with a length of approximately $39 \mu \mathrm{m}$ in each dimension. This corresponds to approximately 14 pixels per wavelength in Perspex or 8 pixels per wavelength in water. These values are both greater than the minimum guideline of 6 pixels per wavelength within the non-destructive testing community [24]. Note, all images in this paper are orientated with the array parallel to the $y$-axis and the image depth along the $z$-axis.

\section{B. Calibration and Test Samples}

To simplify the imaging system, two solid cylindrical Perspex samples were acquired with dimensions of $300 \mathrm{~mm}$ length and a $40 \mathrm{~mm}$ diameter. The specific acoustic impedance, $Z$, of Perspex is $3.5 \mathrm{MPa} \cdot \mathrm{s} \cdot \mathrm{m}^{-1}$ and its speed of sound is $\nu=2720 \mathrm{~ms}^{-1}$, therefore Perspex exhibits similar acoustic properties to water $\left(Z=1.5 \mathrm{MPa} \cdot \mathrm{s} \cdot \mathrm{m}^{-1}\right.$ and $\left.\nu=1500 \mathrm{~ms}^{-1}\right)$ and so can be used to model the acoustic interaction observed in water. Note that since the ultrasonic wavelength in Perspex is approximately twice that in water, the results corresponding to the Perspex samples show a conservative estimate of the sizing accuracy expected in a water load. A flat surface was machined onto the Perspex samples so the phased array could be directly coupled, where the distance from the flat edge to the back wall was $30 \mathrm{~mm}$. One sample was used for calibration purposes, hereafter named the Perspex calibration sample, that was initially defect-free while the second contained an assortment of suspended bubbles that were used for testing purposes, hereafter named the Perspex test sample.

The Perspex calibration sample was modified by drilling five holes, shown in Fig. 1, ranging from 1 to $5 \mathrm{~mm}$ in diameter with $1 \mathrm{~mm}$ increments, into an edge perpendicular to the flat edge at a depth of $15 \mathrm{~mm}$. The side-drilled holes (SDH) were spaced $75 \mathrm{~mm}$ apart to mitigate interference due to scattering from adjacent SDHs. When the phased array was mounted to this Perspex sample, the SDHs were equivalent to twodimensional bubbles relative to the image plane. The Perspex test sample had identical acoustic properties to the Perspex calibration sample except it contained bubbles suspended within. The size distribution of these bubbles was not known and needed to be determined experimentally. This procedure is described in detail in Section IV.

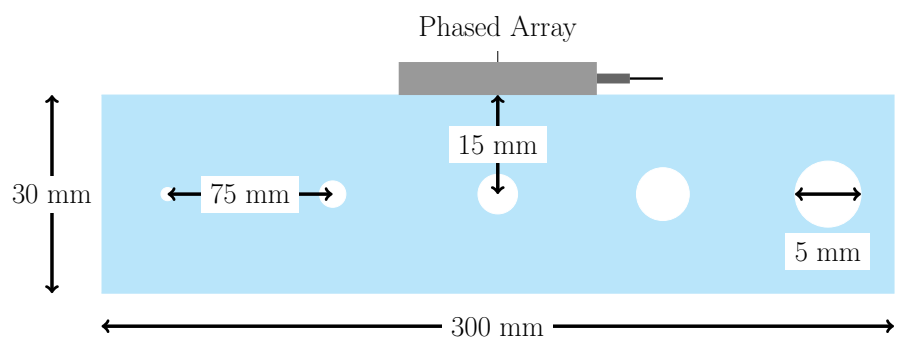

Fig. 1: Schematic of the Perspex calibration sample.

\section{Assessment of Reflector Spatial Variation}

When the array aperture is centred over the SDHs of the Perspex calibration sample, this represents the ideal reflector position because it creates a line of symmetry through the aperture such that spatial variations in the data used to construct the image are minimised. However, when imaging a dynamic industrial process, these reflectors will be positioned randomly throughout the image scene. Therefore, it was important to assess the sizing accuracy as a function of the spatial 


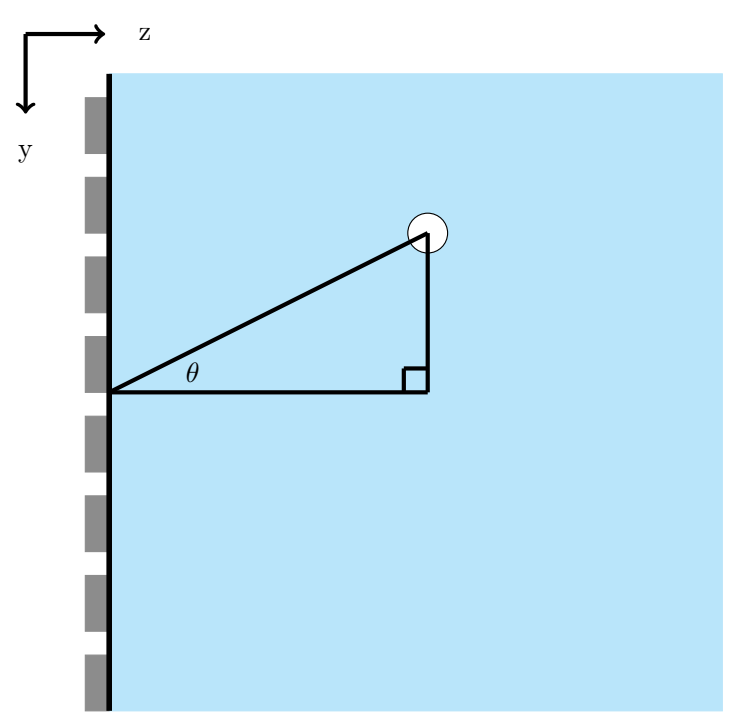

Fig. 2: Schematic of the reflector angle, $\theta$.

orientation of the object relative to the array. To quantify this spatial variation, the reflector angle, $\theta$, has been defined as the angle between the normal to the front face of the array and the line intersecting the centre of the aperture and the centre of the SDH, shown in Fig. 2.

In the calibration setup, the full 128 elements available were used and three FMC data sets were acquired of each SDH of the Perspex calibration sample. These FMC data were sub-sampled using the A-Scan time domain signals corresponding to 32 element apertures. This was achieved using signals corresponding to elements $1-32$, then $2-33$ and so on resulting in $97(128-32+1)$ individual FMC data sets, each representing a discrete reflector angle. This provided the maximum number of possible reflector angles to be investigated using a 32 element aperture. These FMC data sets were then constructed into TFM images under the same conditions except the image scene was shifted along the $y$-axis to ensure the object was kept within the image.

\section{Modelling the Dynamic System}

To model the dynamic nature of the image target it was proposed to move the phased array relative to the position of stationary reflectors. This provided a feasible way to control the relative movement of the reflector with respect to the transducer and enhanced its repeatability. To do this, the phased array was mounted to a six-axis programmable robotic arm (KR 6 R900 sixx, Kuka, Germany), shown in Fig. 3, that scanned both Perspex samples at speeds of 50, 100, $200,300,400$ and $500 \mathrm{mms}^{-1}$. The path of the robotic arm was programmed prior to scanning the Perspex samples to maintain consistency between measurements, where the FMC acquisition was programmed to coincide with the movement of the robot.

When performing FMC, the elements in the aperture are transmitted sequentially but the TFM image scene (the spatial position of the pixels) is set relative to the position of the array elements during the first transmit event. The TFM

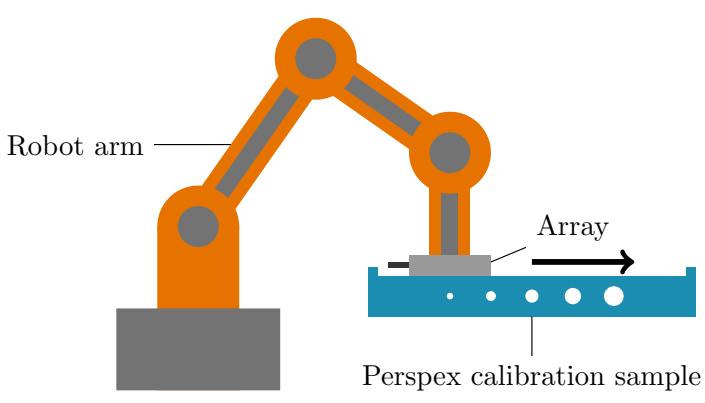

Fig. 3: Robotic arm used to scan Perspex samples at set speeds.

algorithm assumes that the image scene does not change between transmit events such that the temporal resolution of the FMC data set is aligned with the first transmit event.

When the robotic arm was used to scan the Perspex samples, the spatial position of the elements were not constant throughout the acquisition time of an FMC data set. This introduced error into the construction of the TFM images from data acquired under these conditions. Note, this source of error was not applicable when the reflector target was dynamic relative to a stationary array because the spatial position of the elements relative to the image pixels is constant for all transmit events.

If the PAC acquires FMC data at the maximum pulse repetition frequency and it is assumed there is no time delay between transmit events, the total time to acquire an FMC data set $t_{F M C}$ is

$$
t_{F M C}=\frac{2 L \cdot n T_{x}}{\nu},
$$

where $\mathrm{L}$ is the furthest path length in the image scene and $n T_{x}$ represents the number of transmit events. For a 32 element aperture, transmitting to a depth of $30 \mathrm{~mm}$ in Perspex, $t_{F M C}=0.7 \mathrm{~ms}$. If the robot scans the array across the sample at $50 \mathrm{mms}^{-1}$, the position of the array will have moved approximately $35 \mu \mathrm{m}$ or $0.15 \%$ of the 32 element active aperture from its initial position during one FMC acquisition. This spatial offset was not considered to be significant because even at the highest speed investigated, $500 \mathrm{mms}^{-1}$, the array will have only moved approximately $1.6 \%$ of the active aperture from its initial position during one FMC acquisition. This error could not be removed from the TFM imaging algorithm without redesigning the imaging algorithm to account for this spatial offset. Moreover, for the images generated in this paper, the largest array movement corresponds to less than one pixel between transmit events.

Similarly, if the array is stationary relative to a bubble that moves at a velocity of $500 \mathrm{mms}^{-1}$, parallel to the front face of the array, the distance the bubble will have moved during one FMC acquisition would be $0.64 \mathrm{~mm}$ or $2.9 \%$ of the active aperture. Therefore, it is not foreseen that the bubble or robotic arm movement compromise the image quality severely. In addition, given the similar degree of image uncertainty between these two scenarios; using the robotic arm provided a good indication of the response from a moving bubble reflector while facilitating repeatable measurements. 


\section{E. Experimental Verification}

The ultrasound system described in this paper was also tested using a dynamic bubble stream. A schematic of the experimental apparatus is shown in Fig. 4. The linear phased array was positioned in a water tank with a bubble diffuser at its base. Air was pumped to the bubble diffuser via a rotameter to ensure a consistent gas flow rate of $800 \mathrm{~mL} \cdot \mathrm{min}^{-1}$. The array was positioned in-situ to the bubble stream and both intrusive and non-invasive data acquisitions were performed. Non-invasive data acquisition was undertaken by attaching a $10 \mathrm{~mm}$ thick Perspex block to the front face of the array, the Perspex was coupled to the array in the water to ensure no air gaps existed between the two. Since the array is designed to be acoustically matched to Perspex, this facilitated non-invasive measurement. Note, the water supplied did not undergo degassing or any other treatment process prior to be used in these experiments and was approximately $20^{\circ} \mathrm{C}$.

A photograph of the bubble stream is shown in Fig. 5. It was observed that bubbles close to the diffuser and the surface of the water were more coalesced than those in the centre of the bubble stream. Therefore, when acquiring FMC data of this system the array aperture was aligned with the area shown in red in Fig. 5 and the front face of the array was positioned within the bubble stream itself, minimising the path length to reflectors and maximising the signal strength reflected from the bubbles. Since the distribution of bubble sizes in this experimental setup could not be easily controlled or precisely repeated, the photographic image processing algorithm described in Section IV was also applied to this system.

\section{Ultrasound Image Processing Algorithm}

\section{A. Image Upload and Segmentation}

An example of an input TFM image and the segmented TFM image are demonstrated in Fig. 6(a) and (b) respectively. The input TFM image must have square pixels and it must be orientated such that the phased array is parallel to the $y$-axis.

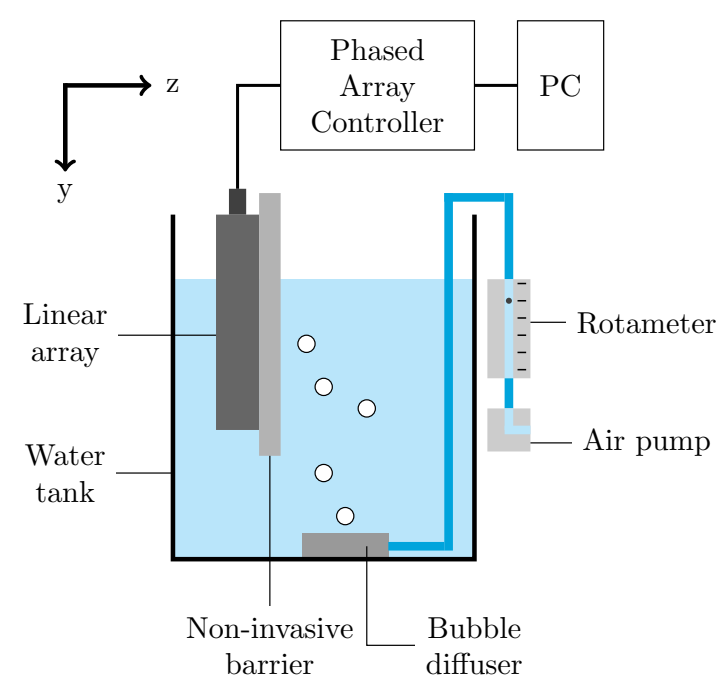

Fig. 4: Schematic representing experimental apparatus to acquire in-situ ultrasonic data of dynamic bubble stream.

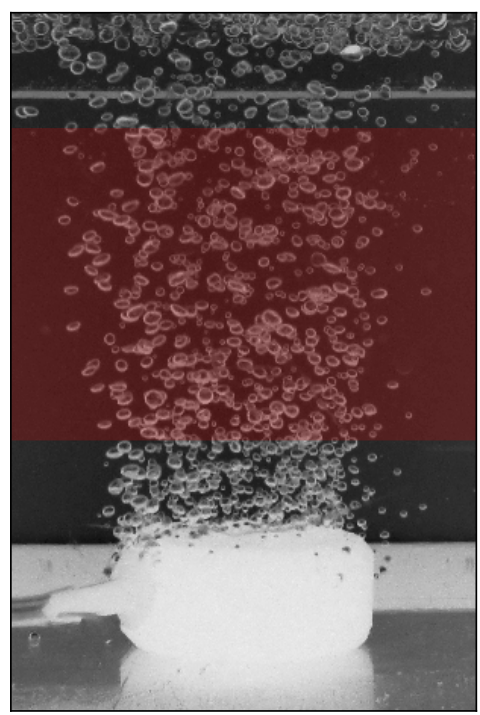

Fig. 5: Photograph of dynamic bubble column, the region of interest is highlighted in red.

The reason for this is that the algorithm expects the movement of bubble objects to be from the base to the top of the image scene.

Image segmentation was used to separate bubble objects located in the foreground of the image scene from the background signal. A global threshold value was determined for each image frame to reduce the computation time of the segmentation process. Yen's method [25] was selected for determination of the segmentation threshold because it is stable for images with a low foreground signal. This is because the method takes the total signal into consideration rather than the inter-class variation, as is the case using Otsu's method [26] for example, that would lead to interference from the high background signal exhibited in these images.

\section{B. Key-point Identification}

The second step of the algorithm is the identification of three key-points on the perimeter of each bubble object in the image. Three key-points are used because this is the minimum number required to model a circular shape. First the coordinates of the perimeter of each object in the binary image are determined. If no contours are found, the image frame is marked as invalid and the algorithm begins processing the next image. The remainder of the algorithm is applied to each individual object in the segmented TFM image. If the coordinates of the object cross the edges of the image frame, the object is not completely within the image frame so this object is marked as invalid and the algorithm begins processing the next bubble object.

The first two key-points are set at the upper and lower bounds of the bubble objects and a third point on the outer circumference between the first two. These key-points are shown in Fig. 6(b) for a $3 \mathrm{~mm}$ diameter SDH in the Perspex calibration sample. 
(a)

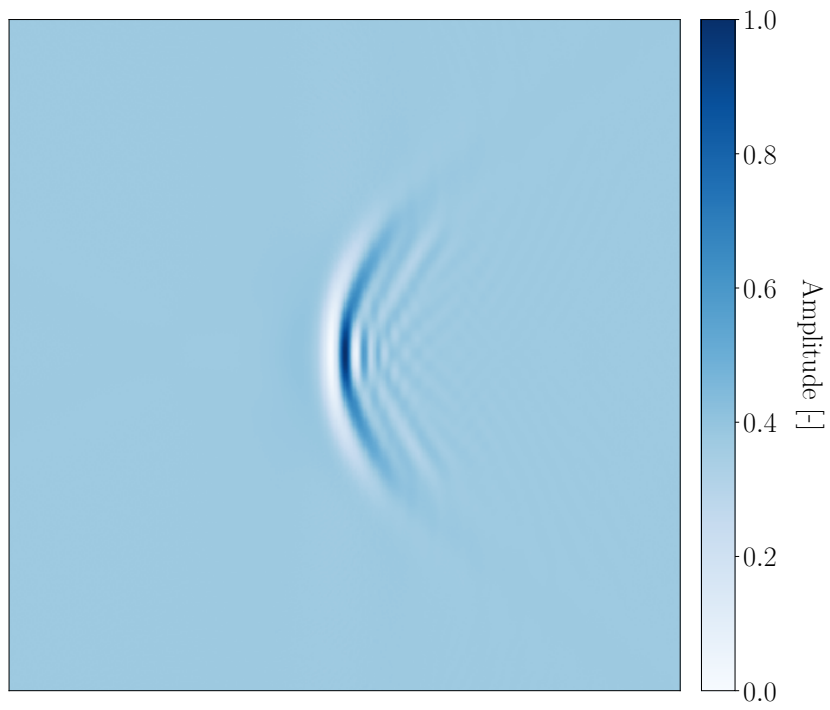

(b)

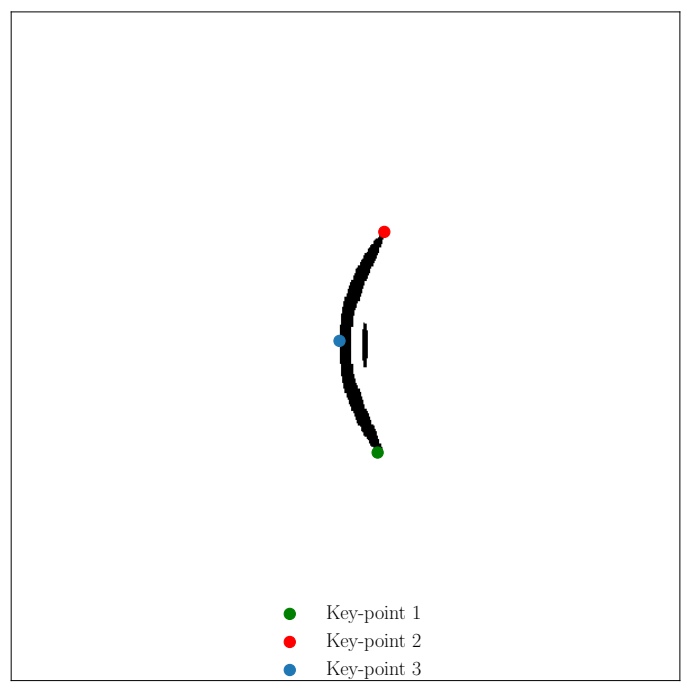

Fig. 6: Images outlining image upload and segmentation processes of the image processing algorithm (a) linear scale TFM image (b) segmented TFM image showing three keypoint positions.

Selection of key-points 1 and 2 requires the algorithm to identify a group of candidate boundary coordinates in the lower and upper halves of the object respectively. The reason for doing this is that the TFM will map the shape of the object depending on its location relative to the centre of the array. In Fig. 6(b), the object is perfectly centred with respect to the centre of the phased array. Whereas, if the object is located at the base or top of the image scene, its curvature could lead to misidentification of the corresponding key-point position. From this group of candidate coordinates the algorithm then determines the correct one, as outlined below, and assigns this as the corresponding key-point.

Key-point 1, shown by the green point in Fig. 6(b), is determined by assessing the boundary coordinates in the lower half of the object. Boundary coordinates on the two right-most column indices of the object are initially selected, shown as the red candidate coordinates in Fig. 7(a) zoomed to the region of interest. From this first group of candidate coordinates, the coordinate with the lowest row index is selected. Next, all boundary coordinates with this same row index are identified, shown as the red coordinates in Fig. 7(b). From this second group of candidate coordinates, the left-most column index is selected as the coordinate position of key-point 1 . The same process is repeated for boundary coordinates in the upper half of the object to determine the coordinate of key-point 2 .

To identify key-point 3, shown by the blue point in Fig. $6(\mathrm{~b})$, the midpoint between key-point 1 and key-point 2 is first determined. Then the equation of the line that passes through key-points 1, 2 and their midpoint is determined, with a gradient, $m_{1}$. The gradient, $m_{2}$, perpendicular to $m_{1}$ was determined from $m_{2}=-1 / m_{1}$. Key-point 3 is then determined from boundary coordinates that intersect this perpendicular line, where the coordinate with the left-most column index is selected to be key-point 3. If the gradient, $m_{1}$ is not defined, then key-point 3 is determined from the line intersecting the midpoint that is parallel to the $z$-axis.

\section{Projection of Circles onto Bubble Objects}

The third step of the algorithm is to fit a circular shape through the three key-points of each bubble object. The procedure to identify the centroid and radius of this circle is shown in Fig. 8. The procedure is to determine the bisector between key-points 1 and 3 and between key-points 2 and 3. The centroid of the circle is determined from the intersect of their perpendicular bisectors. The radius is then calculated using the distance between the centroid and any one of the key-points.

In Fig. 8 a smaller secondary object can be observed behind the larger object. This is an artefact of the ultrasound image and does not represent the location of a real reflector. Therefore, following identification and sizing of all objects in the image, any objects corresponding to these artefacts are identified and marked as invalid. The procedure for this is to determine the Euclidean distance between the centroids of all sized objects. Then where an object is located within the area of second object, if the sum of its radius and the Euclidean distance to the second object is smaller than the radius of the second object, the second object is marked as invalid. Finally, individual objects can be marked as invalid if they have a radius outwith user-defined limits.

\section{Photographic Verification Algorithm}

Alongside the image processing algorithm in Section III, a second image processing algorithm was created to determine the BSD from photographs. Photographic analysis was selected to verify the ultrasonic image analysis because it is already well-established within the process analysis community and it provides comparable spatial information about the system. In addition, given that the BSD in the Perspex test sample and in the dynamic bubble stream were not known, 
(a)

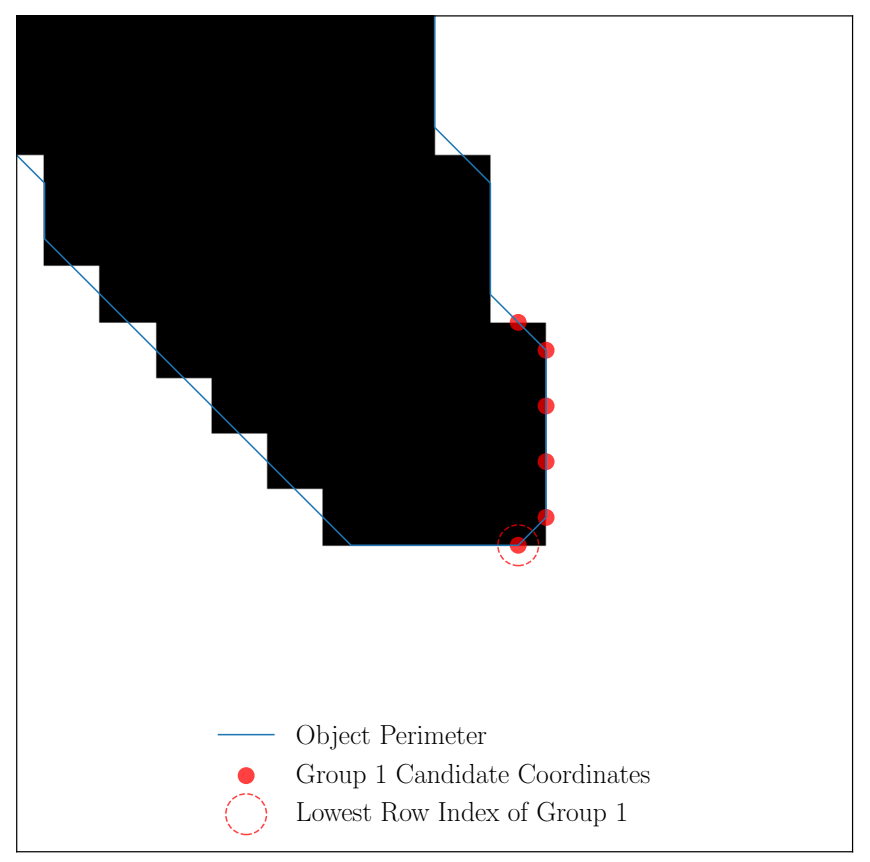

(b)

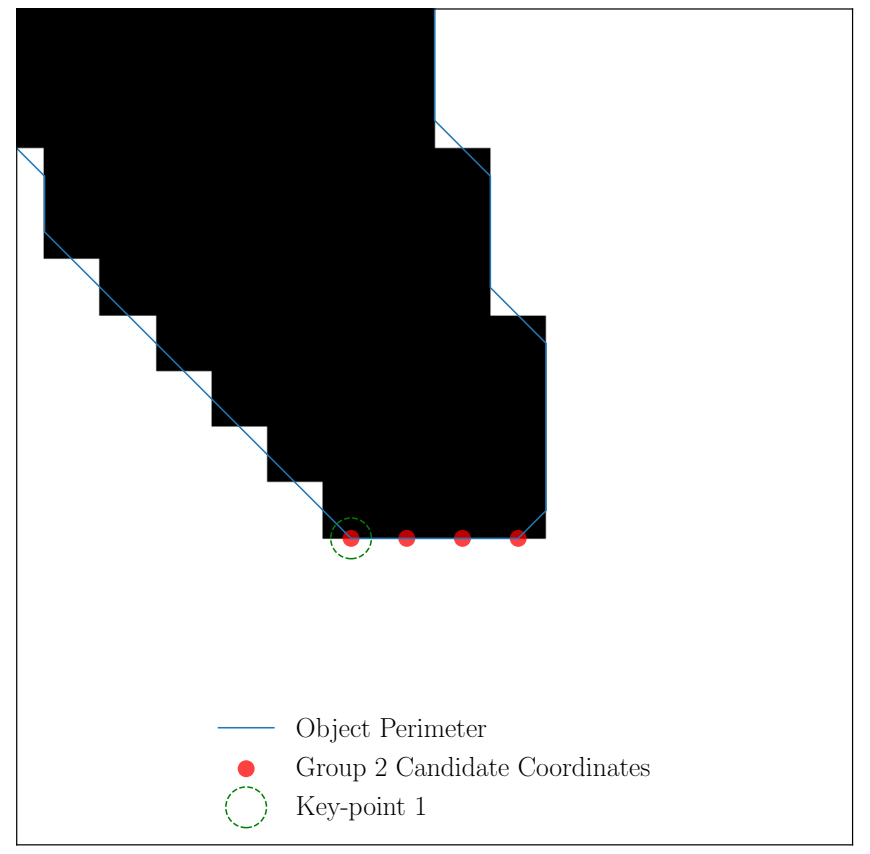

Fig. 7: Key-point identification procedure for key-point 1 showing segmented image zoomed to region of interest (a) group 1 candidate coordinates and lowest row index from this group and (b) group 2 candidate coordinates and location of key-point 1.

this analysis provides ground truth for the ultrasound image processing algorithm.

Photographs were captured using a digital camera (Nikon, Japan) with a 20.7 mega pixel resolution. The pixel dimension

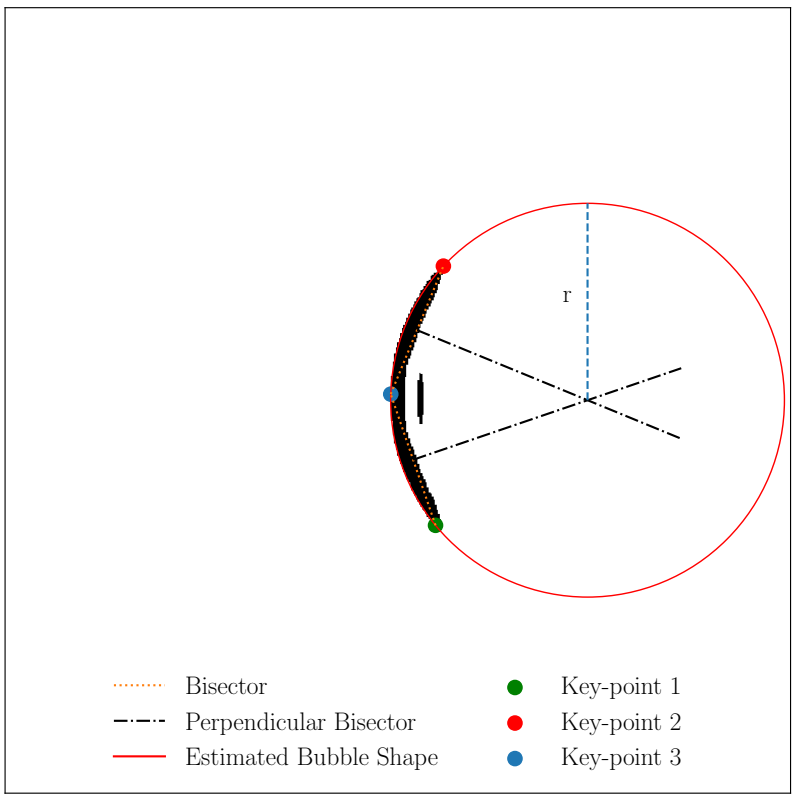

Fig. 8: Technical diagram showing properties used to determine centre of circle and radius, $r$, from three points.

was estimated by positioning a ruler behind the Perspex test sample and gridded paper behind the dynamic bubble stream. The region of interest was illuminated using an LED lamp with an illuminated area of $200 \times 210 \mathrm{~mm}^{2}$ and an illumination intensity of $10 \mathrm{klx}$, positioned behind the image target. Photographs were exported in .jpg format to a PC to be read into the image processing algorithm.

The three-channel RGB images were converted to greyscale using skimage.color.rgb2grey [27] and cropped to reveal just the bubbles relating to the region of interest, shown in Fig. 9(a). Image segmentation is then performed using a global threshold value determined by Otsu's method [26]. The image segmentation resulted in several regions not being closed or well-defined, so binary dilation was applied using an arbitrarily chosen $16 \times 16$ pixel structure to close gaps within objects. This was followed by binary hole filling and binary erosion using the same pixel structure where the result is recorded in Fig. 9(b).

The Watershed algorithm [28] was used to segment the individual bubble objects in the photographs. The Watershed algorithm first identifies markers in the binary image corresponding to local maxima relative to the distance to the background of the image. These markers represent the basins to be filled by the Watershed algorithm. The second stage of the Watershed algorithm is equivalent to filling the image from these marker positions, where the edge of a region is defined as the position where the watershed to a neighbouring region is reached. The result of application of the Watershed algorithm was recorded in Fig. 9(c), where the perimeters of the identified objects have been recorded by the red lines.

The area of the individual regions were converted into circles with the same area and the equivalent diameter $(\mathrm{mm})$ of these circles was used as the estimate of the bubble diameter. 
(a)

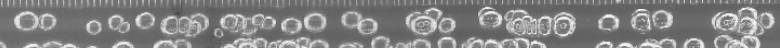

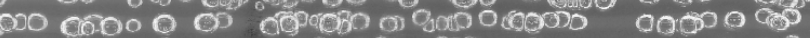

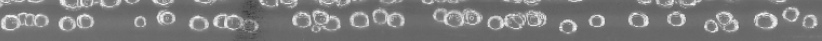

(b)

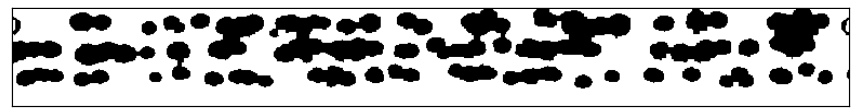

(c)

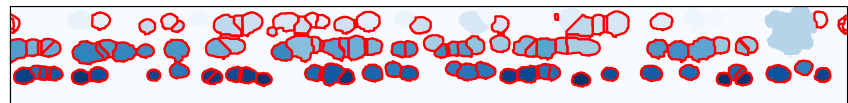

Fig. 9: Photographic image processing stages of Perspex test sample (a) greyscale image including millimetre scale (b) segmented image, scale has now been cropped from image (c) corresponding image after Watershed segmentation, regions of interest are highlighted by red contours.

User-defined diameter limits were determined from manual inspection of small and large bubbles in the images that were clearly separated from other bubbles.

Three photographs were captured along the length of the Perspex test sample to ensure maximum coverage of the bubble reflectors. The lower and upper diameter limits measured from manual inspection were approximately $2.0 \mathrm{~mm}$ and 5.5 $\mathrm{mm}$ respectively. The equivalent diameters were collated into a single array and recorded as a histogram in Fig. 10. It was observed from the empirical data that the BSD was described by a Normal distribution, where the mean and standard deviation of the distribution were $3.4 \pm 0.8 \mathrm{~mm}$ respectively (sample size $n=276$ bubbles) and its probability density function (PDF) has been superimposed onto the histogram.

Five photographs were captured of the dynamic bubble stream and these were cropped to the red region in Fig. 5. From these the lower and upper diameter limits were measured to be approximately $0.5 \mathrm{~mm}$ and $4.5 \mathrm{~mm}$ respectively. The equivalent diameters of the bubbles within these limits were recorded in the histogram in Fig. 11, where the mean and standard deviation were $1.8 \pm 0.8 \mathrm{~mm}$ ( $n=597$ bubbles $)$ and the corresponding PDF has been superimposed onto the histogram. Note, there were fewer bubbles recorded in the photographs of the Perspex test sample than in the dynamic bubble stream, meaning there is increased certainty in the distribution of the dynamic bubble stream than the Perspex test sample.

\section{RESULTS}

\section{A. Impact of Image Quality}

TFM images of the five SDHs in the Perspex calibration sample were passed to the ultrasound image processing algorithm. When the estimated shape and position of the SDHs is superimposed onto the segmented TFM image, for example the red dashed circle in Fig. 12, it was observed that using three key-points was sufficient to model the curvature of the

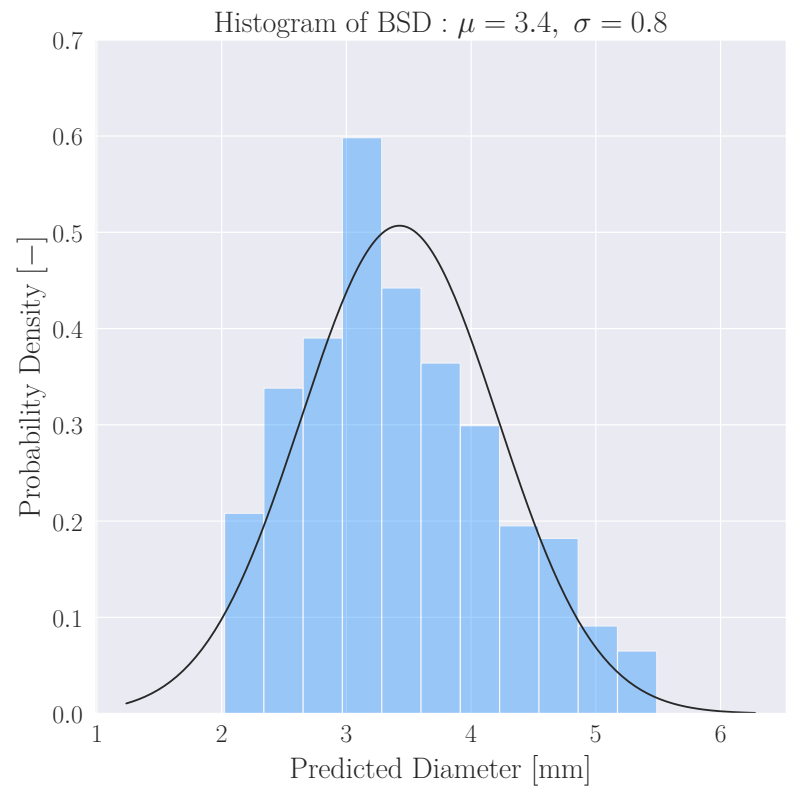

Fig. 10: BSD estimated from photographs of Perspex test sample ( $n=276$ sized bubbles) with the Normal PDF fitted to the empirical data.

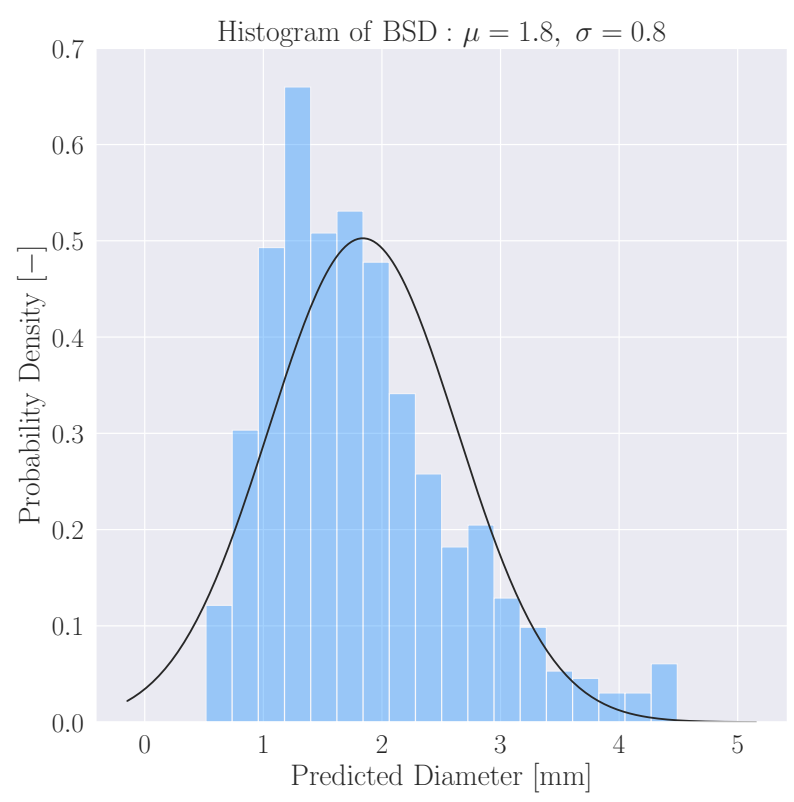

Fig. 11: BSD estimated from photographs of dynamic bubble column ( $n=597$ sized bubbles) with the Normal PDF fitted to the empirical data.

reflectors. In addition, when the true diameter and position of the SDH were superimposed onto the same image, shown by the green solid circle in Fig. 12, the predicted shape is shown to closely represent the true shape. However, this also highlights that the sizing error depends on the representativeness 


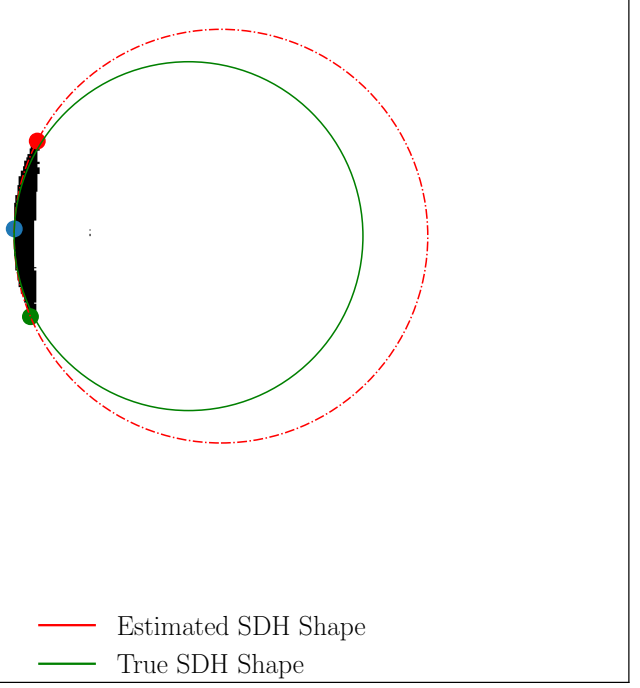

Fig. 12: Segmented TFM image of a $2 \mathrm{~mm}$ diameter SDH in Perspex where the red dashed line represents the shape of the SDH estimated by the image processing algorithm and the green solid line represents the shape of the SDH corresponding to its know radius.

of the segmented TFM image rather than the robustness of the image processing algorithm.

\section{B. Sizing Accuracy of Stationary Reflectors}

The predicted diameter of each SDH in the Perspex calibration sample was recorded as a percentage error of the true value in Fig. 13. These have been recorded as a mean and standard deviation value for each SDH where the error bars represent one standard deviation across the three TFM image replicates.

The error bars in Fig. 13 indicate that as the reflector diameter increases, the SDHs are more consistently mapped onto the TFM image, therefore enabling more precise diameter prediction for larger reflectors. Similarly, it is observed that the average error of the five SDHs decreases as the diameter increases. This is because larger reflectors generate a stronger reflection at the Perspex-air acoustic boundary such that the corresponding segmented TFM image more closely represents the true shape of the reflector.

From Fig. 13 it is observed that the algorithm breaks down for reflectors smaller than four wavelengths in diameter. However, given that typical industrial processes such as solvent sublation or coal liquefaction require bubbles to have a diameter larger than this threshold, this is not foreseen to present a problem for practical deployment of the sizing algorithm [10] [12]. Indeed, the SDHs with diameters greater than four wavelengths exhibited a relative error within $10 \%$. Objects below the four-wavelength threshold were still successfully

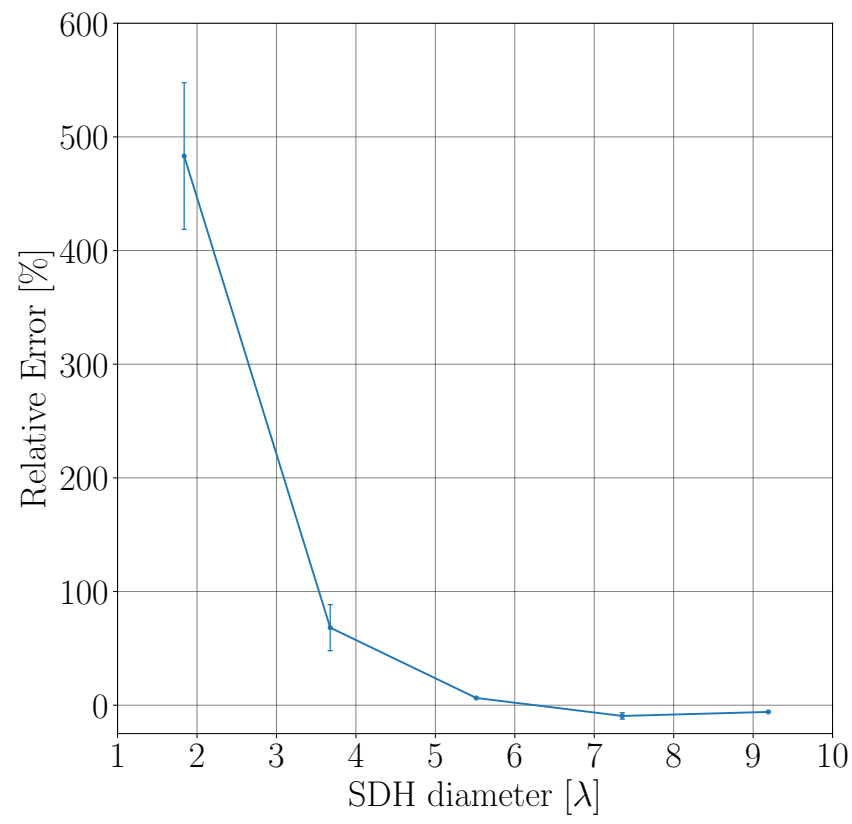

Fig. 13: Relative error of predicted SDH diameters from three TFM images of each SDH in Perspex calibration sample with the aperture centered over each SDH that is stationary relative to the reflector positions.

segmented from the TFM images, however, the reduced sizing accuracy was due to elongation of the segmented objects resulting from the TFM not adequately mapping the curvature of these objects onto the image scene.

\section{Impact of Reflector Spatial Variation}

The TFM images generated from 32 element sub-apertures of the 128 element FMC data sets were passed to the sizing algorithm and the results were recorded as a mean and standard deviation at each angle in Fig. 14. For the TFM image dimensions considered in this work, the maximum reflector angle possible is approximately $\pm 25^{\circ}$ relative to the centre of the aperture, therefore, only the image frames with reflectors within this range have been recorded. The results were consistent with those in Fig. 13; the larger the SDH diameter the greater the accuracy of the diameter prediction and the more precise the result, indicated by the decreasing size of the error bars. Note, the points at $0^{\circ}$ in Fig. 14 are the same values shown in Fig. 13. For SDHs larger than four wavelengths in diameter it was found that the reflector angle does not generate spurious results and the average accuracy across all angles was within $10 \%$. Therefore, the algorithm was shown to be robust enough to cope with spatial variations for large reflectors.

\section{Modelling Dynamic Reflectors}

The robotic arm was used to scan the Perspex calibration sample at the velocities recorded in Section II-D while continuously acquiring FMC data. Upon sizing of the SDHs in each TFM image, the relative error was recorded as a mean 


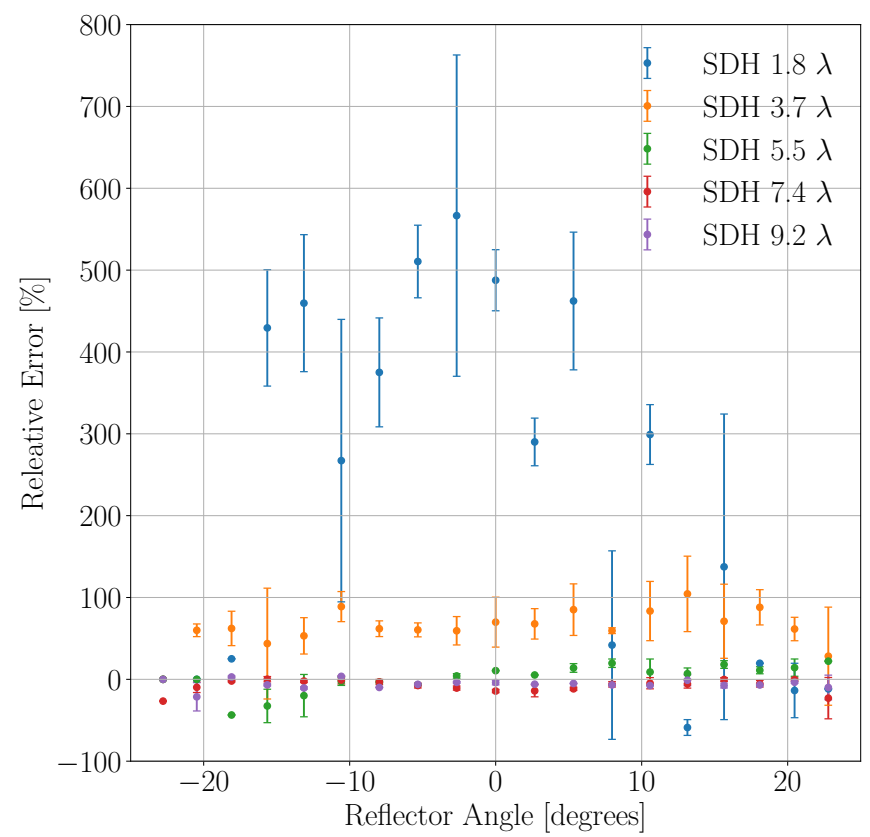

Fig. 14: Relative error of predicted SDH diameters from three TFM images of each SDH in Perspex calibration sample acquired at range of reflector angles under stationary conditions.

and standard deviation at each velocity in Fig. 15, where the error bars correspond to one standard deviation. Note, there were fewer FMC data sets recorded at faster velocities, so the mean value has a lower sample size at faster velocities.

The results from the dynamic system in Fig. 15 are consistent with those from the stationary system in Figs. 13 \& 14; the larger the SDH diameter the greater the accuracy and precision of the diameter prediction. Indeed, for the largest SDH diameter investigated, the algorithm was robust enough to size the SDH within $10 \%$ at a speed of $500 \mathrm{mms}^{-1}$. This shows that the acquisition rate of the ultrasonic data was high enough to generate images that were representative of the shape of the reflector. Also, for SDHs greater than $4 \lambda$ in diameter, the algorithm was able to estimate the SDH diameter within $45 \%$ for velocities up to $300 \mathrm{mms}^{-1}$. Overall, these results indicate, in agreement with the stationary acquisition, that the sizing algorithm is robust under controlled dynamic conditions for reflectors larger than $4 \lambda$ in diameter.

Following this, the sizing algorithm was applied to TFM images of the Perspex test sample acquired under dynamic conditions. As recorded in Section IV, the Perspex test sample contained bubble reflectors ranging from $2.0(3.7 \lambda)$ to $5.5 \mathrm{~mm}$ $(10.1 \lambda)$ in diameter. Also, from Fig. 15 it is observed that for diameters greater than $3.7 \lambda$, the sizing accuracy deteriorates above $300 \mathrm{mms}^{-1}$. Therefore, the robotic arm was used to scan the Perspex test sample at $300 \mathrm{mms}^{-1}$ and a total of 100 FMC data sets were recorded. An example of a segmented and annotated TFM image generated under these dynamic conditions is recorded in Fig. 16. Reflectors not sized in Fig. 16 were either outwith the size limits or too close to the edge of the image so were marked as invalid. Given that Fig. 16 corresponds to the array moving at $300 \mathrm{mms}^{-1}$ it

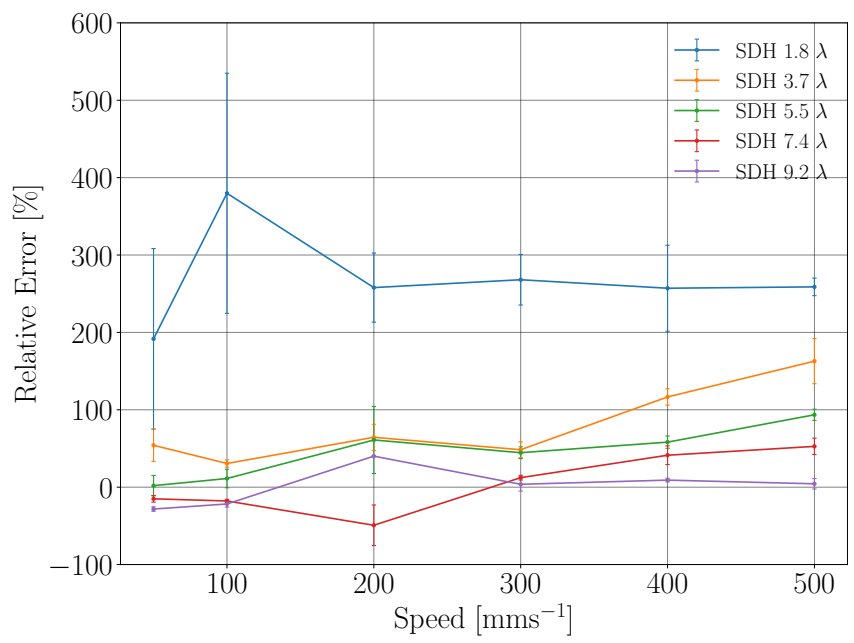

Fig. 15: Relative error of predicted SDH diameters from TFM images, acquired with the array mounted to robotic arm and scanning the Perspex calibration sample at different velocities.

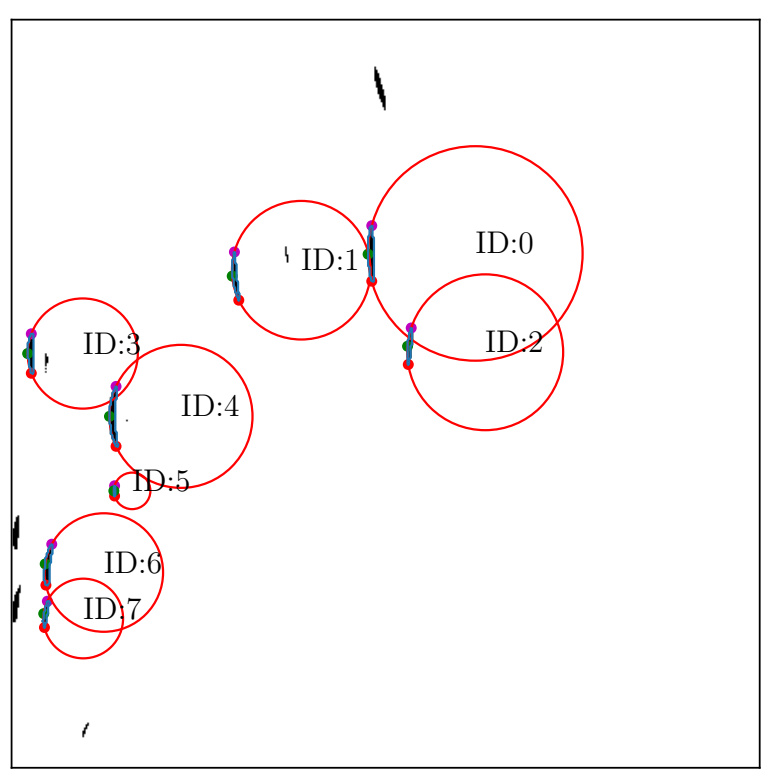

Fig. 16: Example segmented and annotated TFM image of Perspex test sample scanned using the robotic arm at 300 $\mathrm{mms}^{-1}$.

is clear the data acquisition rate is high enough to prevent image distortion, however, this movement is only parallel to the array. When measuring the bubble stream in water, there is interaction between adjacent bubbles and the bubble path is not linear, so the dynamic nature of the result shown in Fig. 16 pertains to a simplified bubble movement.

There were $n=270$ valid bubble reflectors sized in the 100 TFM images of the Perspex test sample. These were recorded as a histogram in Fig. 17, where the size limits were set to the same values in Section IV and a Normal PDF has been fitted to the empirical data. This result is in close agreement with that of the photographic analysis of the Perspex test 


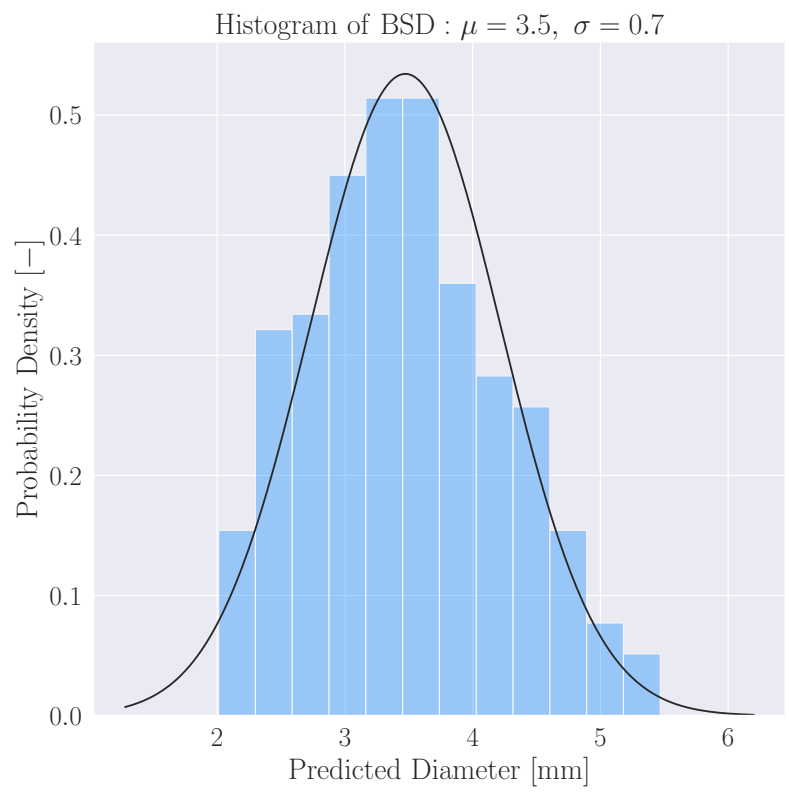

Fig. 17: Histogram of BSD recorded from TFM images of Perspex test sample scanned with the robotic arm at 300 $\mathrm{mms}^{-1}$ ( $n=270$ sized bubbles) with the Normal PDF fitted to the empirical data.

sample. In Fig. 10, the empirical mean and standard deviation of the BSD in the Perspex test sample were $3.4 \pm 0.8 \mathrm{~mm}$. Similarly, Fig. 17 shows the empirical mean and standard deviation were $3.5 \pm 0.7 \mathrm{~mm}$. This provided certainty that the BSD recorded from the ultrasound image processing algorithm provided a relatively accurate measure, especially given the sample sizes from the two workflows were within 3\% of each other. However, given that the exact diameter of each bubble suspended in the Perspex test sample was not known, it was not possible to provide an absolute measure of the certainty of the ultrasound image processing algorithm.

\section{E. Application to Dynamic Process Stream}

One hundred FMC data sets were acquired of the bubble stream in water with the array positioned intrusive to the process and non-invasively via a $10 \mathrm{~mm}$ Perspex barrier mounted to the front face of the array. Examples of a segmented and annotated TFM image acquired under these two conditions were recorded in Fig. 18. In both examples, the reflectors are much smaller than those in Fig. 16. Although the bubbles are larger with respect to the ultrasonic wavelength in water, the signals appear weaker due to increased bubble density leading to scattering and masking of the ultrasonic energy. In addition, given that the acoustic impedance mismatch between Perspex and air is greater than between water and air, the total energy reflected back to the transducer was less.

A study by Aybers and Tapucu [29] has shown millimetre length-scale bubbles rising through a stagnant fluid from a low initial velocity exhibit a rectilinear velocity of approximately
$300 \mathrm{mms}^{-1}$ and this is reached within the first $10 \mathrm{~mm}$ from bubble evolution. Here, the bubble velocity was not known and they were generated under pressure from the air pump so it is likely that the bubbles were travelling much faster than $300 \mathrm{mms}^{-1}$ and their velocities were outwith the scope of this investigation. Indeed, as the bubble velocity increases relative to $t_{F M C}$, the object would become elongated as it is mapped onto the TFM image, reducing its representativeness. Given this complex dynamic nature and the interaction between bubbles, the results obtained could not be easily repeated or verified. Note, the non-invasive image in Fig. 18(b) corresponds to a particularly good image result, with several reflectors in the image scene. However, across the 100 image frames the average number of reflectors per TFM image was approximately 0.62 when acquired non-invasively and 2.86 when acquired intrusively. Therefore, a high number of FMC acquisitions are required to establish an accurate distribution of the bubbles sizes.

The estimated bubble sizes from the intrusive and noninvasive imaging were recorded in Figs. 19(a) and (b) respectively. From the previous analyses of stationary and dynamic reflectors, it can only be said that objects greater than approximately $4 \lambda$ can be sized within $10 \%$ certainty. In water, this corresponds to bubbles with a diameter greater than $1 \mathrm{~mm}$, therefore, the lower size limit of the diameters recorded in the BSD histogram is set to $1 \mathrm{~mm}$ rather than $0.5 \mathrm{~mm}$ used for the photographic analysis.

The histograms in Fig. 19 both show a mostly uniform distribution across the sample bins, however, from Fig. 11 it was shown to follow a Normal distribution. Given that the sample size in Fig. 19(b) is only 62, about $10 \%$ of that relating to Fig. 11, the 'true' PDF relating to ultrasound imaging system is unclear due to under sampling. The reason for this discrepancy is the lower image quality due to the complexity of the bubble stream. However, these results do indicate that the image processing algorithm presented in this paper can be deployed to generate a measure of the BSD both invasively and non-invasively.

\section{DISCUSSION}

In this paper, an algorithm for the estimation of BSD is presented and evaluated under stationary and dynamic imaging conditions. The accuracy of bubble diameter prediction is found to be sensitive to the accuracy of input images to represent the true shape of the reflectors. In light of these results, this discussion will focus on how the ultrasound system could be modified to enhance the quality of the input images and therefore the accuracy of BSD determination.

The first aspect to consider is the array transmission sequence deployed, where a compromise exists between the frame rate and the signal-to-noise ratio (SNR). This has been specifically addressed in the medical community for cardiac imaging, where high temporal resolution is required to monitor acute conditions [1]. High frame rate imaging is often achieved using parallel beamforming whereby multiple foci are generated in a single transmission event or diverging waves are used that insonify a larger area in a single transmit event 


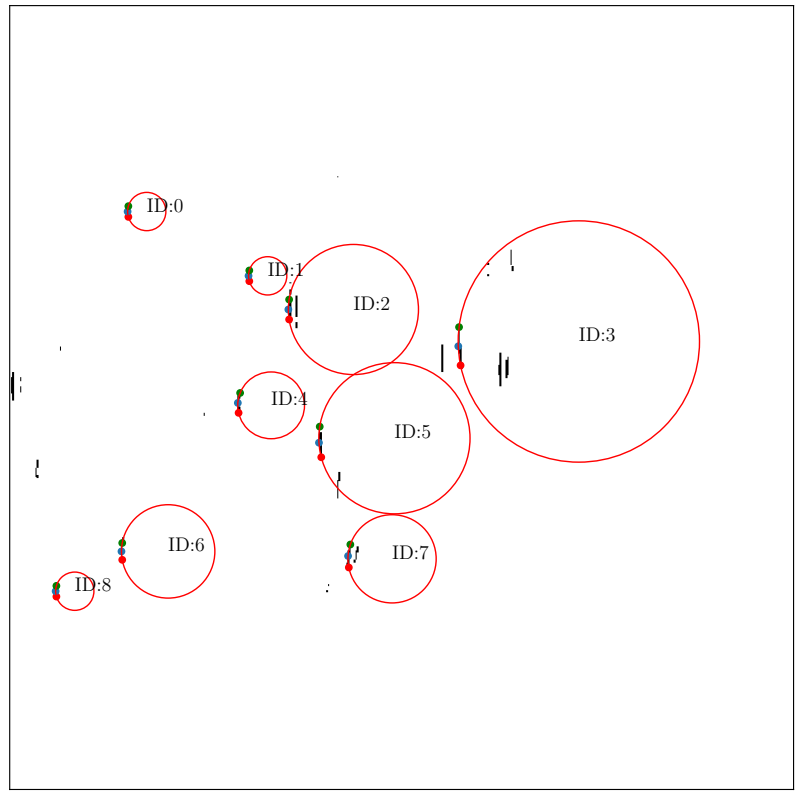

(b)

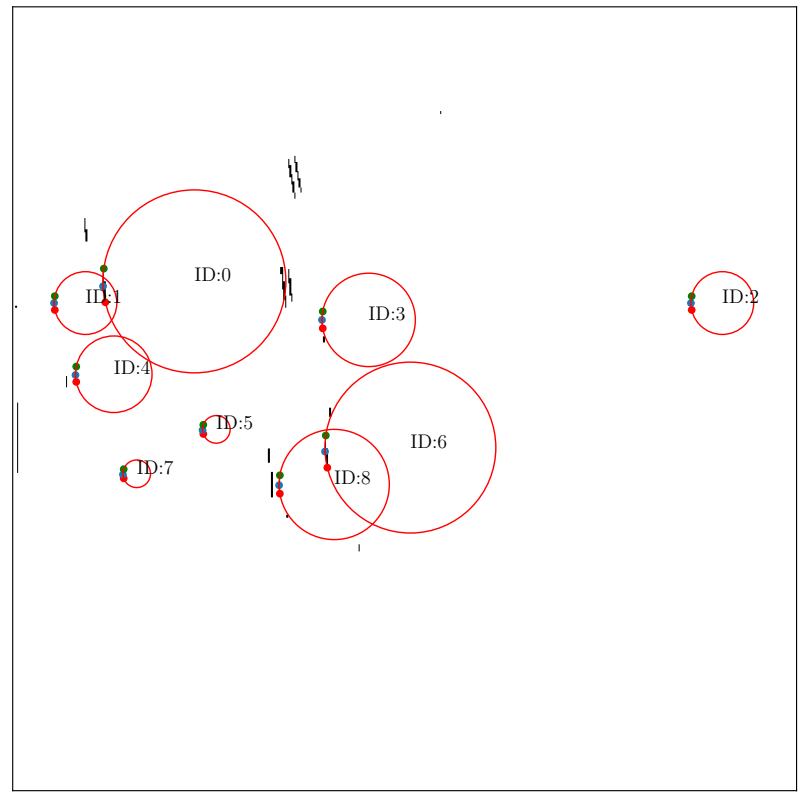

Fig. 18: Example of segmented and annotated TFM images of dynamic bubble stream acquired (a) intrusive to the process and (b) non-invasive to the process via $10 \mathrm{~mm}$ of Perspex.

[4]. A consequence of both approaches is a lower energy in the focal region with respect to using the full aperture to generate a single focus. On the other hand, with respect to FMC, where only a single element is fired during a given transmit event, the energy injected into the load medium is much greater whilst this facilitates frame rates greater than $100 \mathrm{~Hz}$ [4]. Therefore, using alternative transmission sequences such as parallel beamforming and diverging wave compounding could enhance the image SNR and therefore the accuracy of BSD (a)

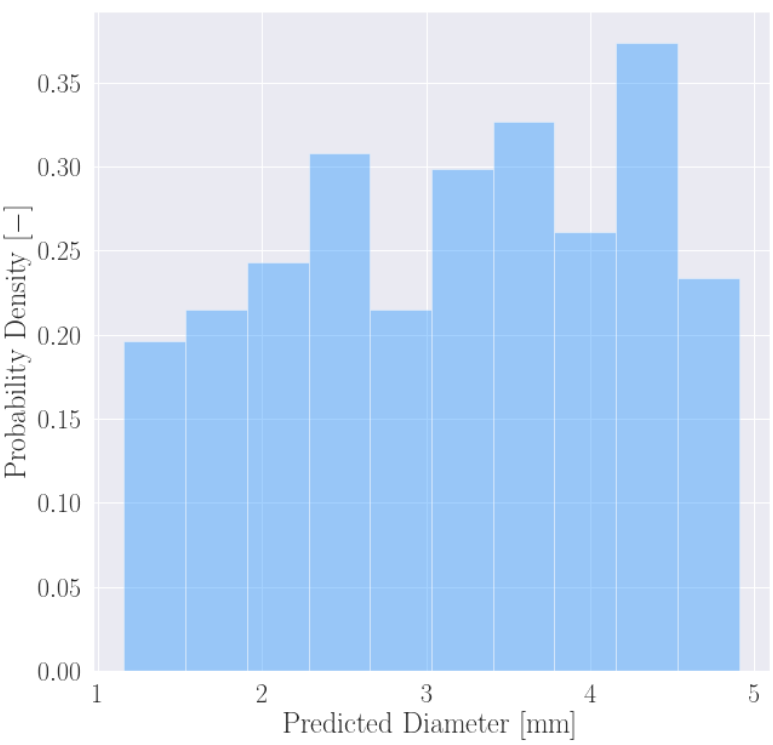

(b)

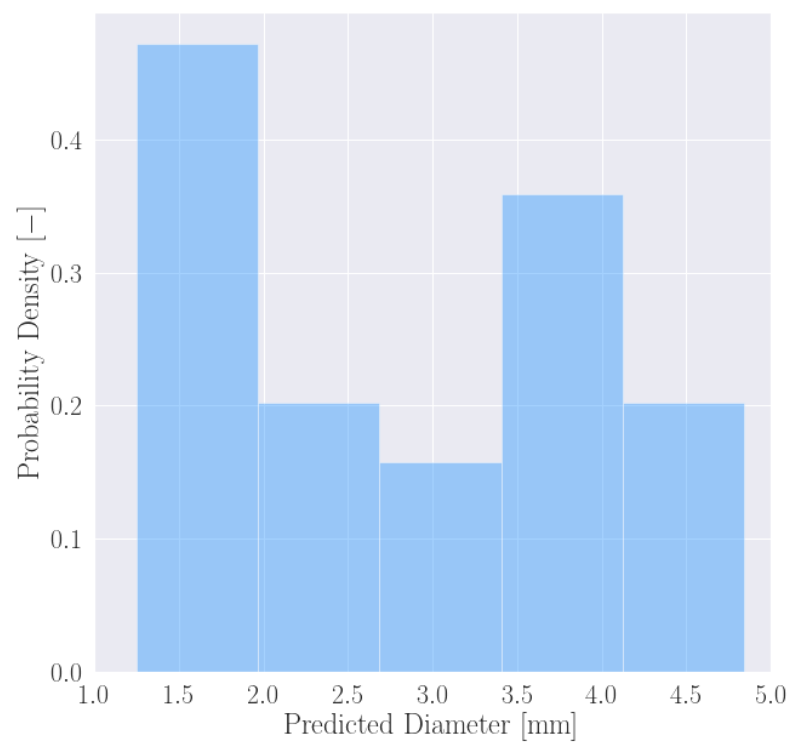

Fig. 19: Histograms of BSD of bubbles rising to surface of water (a) intrusive to the process ( $n=286$ sized bubbles) and (b) non-invasive to the process ( $n=62$ sized bubbles) through Perspex.

determination.

Given the system presented in this paper, increasing the aperture above 32 elements would increase the total information captured within a given TFM image. It is expected that this would lead to better representation of the bubble curvature in the TFM images, enhancing the accuracy of BSD 
determination. However, the active aperture was limited by the number of channels available on the PAC so could not be increased here.

Within the context of industrial process analysis, an advantage of using ultrasonic phased array imaging over ultrasonic tomography is that the path length of the ultrasonic wave is not set by the diameter of the process vessel but instead is userdefined corresponding to the desired image depth. This means that frequency dependent attenuation is no longer a limiting factor in the experimental design, such that higher frequencies can be deployed. Using high-frequency phased array imaging leads to greater lateral resolution creating the possibility for ultrasound imaging of individual reflectors as demonstrated in this paper rather than the bulk system. An implication of reducing the depth-of-field is that the bulk process may be positioned relatively far from the vessel wall, increasing the ultrasonic path length, leading to image deterioration. However, phased array imaging could be deployed alongside ultrasonic tomography to provide complementary information relating to the bulk and peripheral process conditions.

In this paper, phased array imaging was applied to a stream of air bubbles rising through water where it was found that the bubble density reduced the signal intensity and therefore the SNR of the TFM images was low. It should be noted that during the development of the ultrasound image processing algorithm it was assumed that the fluid process medium does not contain scattering particles. If scatterers are present in the process fluid, this leads to image deterioration, compromising the accuracy of BSD determination. The presence of scatterers is typical of biomedical ultrasound imaging where techniques such as phase coherence imaging can be deployed to weight the pixel values corresponding to the energy ratio between the main lobe and side lobe artefacts [30]. This has the impact of reducing the intensity of signals corresponding to scatterers while maintaining the signal intensity from mainbeam reflections, so could be used to overcome this issue.

A specific challenge to perform ultrasound array imaging of industrial processes is the transmission of ultrasound energy through the vessel wall into the process. This has been addressed in this paper using a Perspex non-invasive material to facilitate the transmission of ultrasound energy. However, a typical industrial plant environment contains steel vessels creating a large acoustic impedance mismatch between the transducer and the process. The presence of such an acoustic barrier reduces the quantity of ultrasound energy reaching the process and introduces reverberation into the received signals. Removing these reverberations can be performed using postprocessing of the received data [31], however, the intensity of the transmitted signal must be large to cope with the signal attenuation.

Overall, there are several challenges associated with the practical deployment of ultrasonic phased arrays for imaging in industrial process analysis. However, addressing these should not present a significant burden to overcome thanks to the breadth of innovation already present within of the ultrasound community. In this context, ultrasonic phased arrays are well-poised as a potential tool within the next generation of process analytical technologies.

\section{CONCLUSION}

An image processing algorithm has been presented for the determination of bubble size distribution in two-phase industrial flows. This algorithm was tested using total focusing method ultrasound images generated using a 32 element, 5 $\mathrm{MHz}$ linear phased array. A solid Perspex sample containing reflectors of known diameter was tested under stationary and dynamic conditions by coupling the phased array to a six-axis robotic arm to scan the sample at known velocities. It was found that for reflectors larger than $4 \lambda$ in diameter the sizing accuracy was within $10 \%$ when images were acquired under stationary conditions. This was also true across the expected spatial variation of reflectors. For reflectors larger than $4 \lambda$ in diameter, the sizing accuracy was within $45 \%$ at velocities up to $300 \mathrm{mms}^{-1}$. However, above this velocity the algorithm deteriorated for reflectors smaller than $9 \lambda$ in diameter. When the ultrasound system was applied to a stream of bubbles rising through water, the system was capable of imaging these reflectors both intrusively and non-invasively, via a $10 \mathrm{~mm}$ Perspex barrier. Upon application of the image processing algorithm to these images, it was found that the high bubble density reduced the signal-to-noise ratio, limiting the sample size in the measured distribution. The bubble sizing accuracy was found to be sensitive to the input image provided. Where the signal-to-noise ratio of these images is dependent on the ultrasonic energy injected into the system and on the scatterer density in the load medium. Overall, this paper shows the high potential for ultrasonic phased array imaging and sizing of two-phase industrial flows both intrusively and non-invasively.

\section{ACKNOWLEDGEMENT}

The authors would like to thank BP Chemicals Limited for their support and industrial direction.

\section{REFERENCES}

[1] M. Cikes, L. Tong, G. R. Sutherland, and J. D'hooge, "Ultrafast cardiac ultrasound imaging: technical principles, applications, and clinical benefits," JACC: Cardiovascular Imaging, vol. 7, no. 8, pp. 812-823, 2014.

[2] A. Karn, C. Ellis, R. Arndt, and J. Hong, "An integrative image measurement technique for dense bubbly flows with a wide size distribution," Chemical Engineering Science, vol. 122, pp. 240-249, 2015.

[3] B. Drinkwater and P. Wilcox, "Ultrasonic arrays for non-destructive evaluation: A review," NDT \& E International, vol. 39, no. 7, pp. 525 $-541,2006$.

[4] L. Tong, H. Gao, H. F. Choi, and J. D'hooge, "Comparison of conventional parallel beamforming with plane wave and diverging wave imaging for cardiac applications: A simulation study," IEEE transactions on ultrasonics, ferroelectrics, and frequency control, vol. 59, no. 8, pp. 1654-1663, 2012.

[5] B. Hoyle, "Process tomography using ultrasonic sensors," Measurement Science and Technology, vol. 7, no. 3, pp. 272 - 280, 1996. [Online]. Available: http://stacks.iop.org/0957-0233/7/i=3/a=007

[6] L. W. Schmerr, "An ultrasonic system," in Fundamentals of Ultrasonic Nondestructive Evaluation. Springer, 2016, pp. 1-13.

[7] M. J. Povey, "Ultrasound particle sizing: A review," Particuology, vol. 11, no. 2, pp. 135-147, 2013.

[8] V. J. Pinfield and M. J. Povey, "A perturbation approach to acoustic scattering in dispersions," The Journal of the Acoustical Society of America, vol. 120, no. 2, pp. 719-732, 2006.

[9] F. Alba, G. Crawley, J. Fatkin, D. Higgs, and P. Kippax, "Acoustic spectroscopy as a technique for the particle sizing of high concentration colloids, emulsions and suspensions," Colloids and Surfaces A: Physicochemical and Engineering Aspects, vol. 153, no. 1-3, pp. 495-502, 1999. 
[10] J. S. Smith, L. F. Burns, K. T. Valsaraj, and L. J. Thibodeaux, "Bubble column reactors for wastewater treatment. 2. the effect of sparger design on sublation column hydrodynamics in the homogeneous flow regime," Industrial \& engineering chemistry research, vol. 35, no. 5, pp. 17001710, 1996.

[11] S. Sie and R. Krishna, "Fundamentals and selection of advanced fischertropsch reactors," Applied Catalysis A: General, vol. 186, no. 1-2, pp. 55-70, 1999.

[12] H. Ishibashi, M. Onozaki, M. Kobayashi, J.-i. Hayashi, H. Itoh, and T. Chiba, "Gas holdup in slurry bubble column reactors of a $150 \mathrm{t} / \mathrm{d}$ coal liquefaction pilot plant process," Fuel, vol. 80, no. 5, pp. 655-664, 2001.

[13] N. Mehrshad and M. Massinaei, "New image-processing algorithm for measurement of bubble size distribution from flotation froth images." Minerals \& Metallurgical Processing, vol. 28, no. 3, 2011.

[14] N. Sadr-Kazemi and J. Cilliers, "An image processing algorithm for measurement of flotation froth bubble size and shape distributions," Minerals Engineering, vol. 10, no. 10, pp. 1075-1083, 1997.

[15] J. Tamminen, E. Lahdenperä, T. Koiranen, T. Kuronen, T. Eerola L. Lensu, and H. Kälviäinen, "Determination of single droplet sizes, velocities and concentrations with image analysis for reactive extraction of copper," Chemical Engineering Science, vol. 167, pp. 54-65, 2017.

[16] D. Ito, H.-M. Prasser, H. Kikura, and M. Aritomi, "Uncertainty and intrusiveness of three-layer wire-mesh sensor," Flow Measurement and Instrumentation, vol. 22, no. 4, pp. 249-256, 2011.

[17] C. Tompkins, H.-M. Prasser, and M. Corradini, "Wire-mesh sensors: A review of methods and uncertainty in multiphase flows relative to other measurement techniques," Nuclear Engineering and Design, vol. 337, pp. 205-220, 2018.

[18] R. Grau and K. Heiskanen, "Visual technique for measuring bubble size in flotation machines," Minerals Engineering, vol. 15, no. 7, pp. 507$513,2002$.

[19] Z. Zhang, "A flexible new technique for camera calibration," IEEE Transactions on pattern analysis and machine intelligence, vol. $22, \mathrm{pp}$. 1330-1334, 2000

[20] C. Holmes, B. Drinkwater, and P. Wilcox, "Post-processing of the full matrix of ultrasonic transmit-receive array data for non-destructive evaluation," NDT \& E International, vol. 38, no. 8, pp. 701 - 711, 2005.

[21] C. Xu and T. Shepard, "Digital image processing algorithm for determination and measurement of in-focus spherical bubbles," in ASME 2014 4th Joint US-European Fluids Engineering Division Summer Meeting. American Society of Mechanical Engineers, 2014.

[22] M. Yang, H. I. Schlaberg, B. S. Hoyle, M. S. Beck, and C. Lenn, "Realtime ultrasound process tomography for two-phase flow imaging using a reduced number of transducers," IEEE transactions on ultrasonics, ferroelectrics, and frequency control, vol. 46, no. 3, pp. 492-501, 1999.

[23] J. Dziewierz, T. Lardner, and A. Gachagan, "A design methodology for 2D sparse NDE arrays using an efficient implementation of refracted-ray TFM," in 2013 IEEE International Ultrasonics Symposium (IUS), July 2013, pp. 136-138.

[24] Zetec Inc., "Fundamental Principles of FMC and TFM Technologies," 2017, [Online; accessed February 5, 2020].

[25] J.-C. Yen, F.-J. Chang, and S. Chang, "A new criterion for automatic multilevel thresholding," IEEE Transactions on Image Processing, vol. 4, no. 3, pp. 370-378, 1995

[26] N. Otsu, "A threshold selection method from gray-level histograms," IEEE Transactions on Systems, Man, and Cybernetics, vol. 9, no. 1, pp. 62-66, 1979.

[27] S. van der Walt, J. L. Schönberger, J. Nunez-Iglesias, F. Boulogne, J. D. Warner, N. Yager, E. Gouillart, T. Yu, and the scikit-image contributors, "scikit-image: image processing in Python," PeerJ, vol. 2, p. e453, 6 2014. [Online]. Available: https://doi.org/10.7717/peerj.453

[28] F. Meyer, "Topographic distance and watershed lines," Signal processing, vol. 38, no. 1, pp. 113-125, 1994

[29] N. Aybers and A. Tapucu, "The motion of gas bubbles rising through stagnant liquid," Wärme-und Stoffübertragung, vol. 2, no. 2, pp. 118128, 1969.

[30] J. Camacho, M. Parrilla, and C. Fritsch, "Phase Coherence Imaging," IEEE Transactions on Ultrasonics, Ferroelectrics, and Frequency Control, vol. 56, no. 5, 2009.

[31] M. Ingram, A. Gachagan, A. J. Mulholland, A. Nordon, and M. Hegarty, "Ultrasonic Array Imaging Through Reverberating Layers for Industrial Process Analysis," in 2018 IEEE International Ultrasonics Symposium (IUS). IEEE, 2018, pp. 1-4. 